\title{
Economic evaluation of sacral neuromodulation in overactive bladder: A Canadian perspective
}

\author{
Magdy M. Hassouna, PhD, MD, FRCSC, FACS, ${ }^{*}$ Hamid Sadri, PharmD MSc ${ }^{\dagger}$
}

"Division of Urology, University of Toronto, Toronto, ON; 'Director Health Economics and HTA, Medtronic of Canada, Toronto, ON

Cite as: Can Urol Assoc J 2015;9(7-8):242-7. http://dx.doi.org/10.5489/cuai.2711

Published online August 10, 2015.

\section{Abstract}

Introduction: Refractory overactive bladder $(\mathrm{OAB})$ with urge incontinence is an underdiagnosed condition with substantial burden on the healthcare system and diminished patient's quality-of-life. Many patients will fail conservative treatment with optimized medical-therapy (OMT) and may benefit from minimally invasive procedures, including sacral-neuromodulation (SNM) or botulinum-toxin (BonT-A). The goal of this study was to estimate the costefectiveness of SNM vs. OMT and BonT-A as important parameters from coverage and access to a therapy.

Methods: A Markov model with Monte-Carlo simulation was used to assess the incremental cost effectiveness ratio (ICER) of SNM vs. BonT-A and OMT both in deterministic and probabilistic analysis from a provincial payer perspective over a 10-year time horizon with 9-month Markov-cycles. Clinical data, healthcare resource utilization, and utility scores were acquired from recent publications and an expert panel of 7 surgeons. Cost data (2014-Dollars) were derived from provincial health insurance policy, drug benefit formulary, and hospital data. All cost and outcomes were discounted at a $3 \%$ rate.

Results: The annual (year 1-10) incremental quality-adjusted life years for SNM vs. BonT-A was 0.05 to 0.51 and SNM vs. OMT was 0.19 to 1.76. The annual incremental cost of SNM vs. BonT-A was $\$ 7237$ in year 1 and $-\$ 9402$ in year 10 and was between $\$ 8878$ and $-\$ 11447$ vs. OMT. In the base-case deterministic analysis, the ICER for SNM vs. BonT-A and OMT were within the acceptable range (\$44837 and \$15 130, respectively) at the second year of therapy, and SNM was dominant in consequent years. In the basecase analysis the probability of ICER being below the acceptability curve (willingness-to-pay $\$ 50$ 000) was $>99 \%$ for SNM vs. BonT-A at year 3 and $>95 \%$ for OMT at year 2 .

Conclusion: SNM is a cost-effective treatment option to manage patients with refractory $\mathrm{OAB}$ when compared to either BonT-A or OMT. From a Canadian payers' perspective, SNM may be considered a first-line treatment option in management of patients with OAB with superior long-term outcomes. Similar to all economic analysis, this study has limitations which are based on the assumptions of the used model.

\section{Introduction}

The International Urogynecological Association/International Continence Society joint report on terminology defined overactive bladder $(\mathrm{OAB})$ as urgency with or without urge incontinence, usually with frequency and nocturia in the absence of proven infection or other pathology. ${ }^{1,2} \mathrm{OAB}$ is categorized in two distinctive types: (1) OAB dry without any incontinence and (2) $\mathrm{OAB}$ wet with urinary incontinence. ${ }^{3}$ About $12 \%$ to $18 \%$ of Canadians live with some form of $\mathrm{OAB}$, with prevalence figures of about $3.1 \%$ in men and $14.7 \%$ in women; however, these figures fail to account for the underdiagnosed problem of incontinence. ${ }^{4-6}$

The pathology of the disorder suggests a disruption of the spinal reflex mechanism that carries sensory signals from autonomic and somatic pathways from the bladder outlet and tonic inhibitory system in the brain. ${ }^{7}$ This disruption creates inhibits the control function of the parasympathetic excitatory outflow of the urinary bladder. ${ }^{5}$

$\mathrm{OAB}$ can have substantial impact on a patient's quality of life, such as decreased work productivity, sexual dissatisfaction, erectile dysfunction, depression, poorer mental health, and lack of sleep. ${ }^{3,8}$ These can adversely affect a person's physical and psychological well-being by limiting daily activities, intimacy, and worsening self-esteem. ${ }^{9}$

The economic burden of $\mathrm{OAB}$ to the Canadian economy is substantial and includes prescription drugs, medical equipment, therapies, physician time, and lost productivity. ${ }^{10}$ The estimated financial impact of $\mathrm{OAB}$ on healthcare expenditure in the United States is about $\$ 9$ billion. The direct cost of OAB in Canada is about $\$ 175$ million; this figure is increased to $\$ 352$ million when accounting for urgency urinary incontinence. ${ }^{9,11}$ The estimated annual cost of OAB per patient to the Canadian health system is about $\$ 11329$ per annum. ${ }^{4,9}$

Different treatment options are available for $O A B$, including lifestyle or behavioural changes, such as pelvic floor strengthening, bladder retraining, and management of daily 
fluid intake. ${ }^{4,12}$ Optimal medical therapy (OMT) typically aims to restore normal function of the bladder storage phase by relaxing the bladder musculature to render it compliant to urine filling. ${ }^{4}$ Several approved medications are used in management of $\mathrm{OAB}$ in Canada including: oxybutynin chloride, tolterodine tartrate, trospium chloride, solifenacin succinate, darifenacin hydrobromide, and fesoterodine fumarate. ${ }^{10}$ These anticholinergic drugs are intended to block the acetylcholine transmitter in the bladder through the muscarinic receptor subtypes in the detrusor muscle. ${ }^{13-15}$ This inhibition disrupts the frequent spontaneous contractions that occur during the bladder filling. ${ }^{11,16} \mathrm{~A}$ newer group of pharmacological agents based on the use of beta 3 agonists, such as myrabegron, have been added to the armamentarium of drugs for $\mathrm{OAB} .^{2}$

There are serious systemic side effects for these anticholinergic drugs. ${ }^{12}$ Consequently, not all patients may be suited or willing to opt for anticholinergic therapy and may prefer other treatments options, such as sacral nerve modulation (SNM) or notulinum toxin (BonT-A) injections inside the bladder muscle. Clinical trials have demonstrated the clinical efficacy of these therapies. ${ }^{17,18}$ For those individuals who are refractory to medications, intravesical BonT-A injections or SNM have shown significant positive results. The use of intravesical BonT-A injections is associated with durable efficacy and improvement in health related quality of life, but requires multiple annual injections and exposes the patient to a risk of urinary tract infection and urinary retention. ${ }^{19}$ On the other hand, SNM is now widely accepted due to its higher level of consistency and efficacy, but it also requires a high degree of skill on the part of the implanting surgeons in addition to having higher upfront cost. ${ }^{20}$ We estimate the cost-effectiveness of therapeutic options of idiopathic OAB using SNM, BonT-A, and OMT.

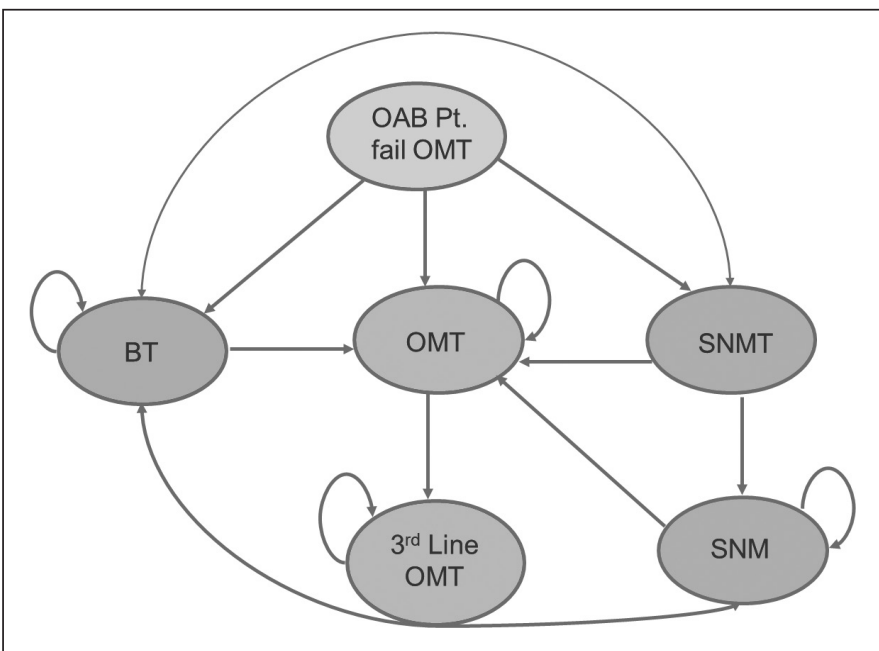

Fig. 1. Markov states.

\section{Methods}

An economic model was used to estimate the long-term cost and outcomes of the above mentioned therapeutic options for OAB from a Canadian provincial health system perspective. The primary outcome measure was quality-adjusted life years (QALY) gained. QALY enabled us to directly compare different therapies. In this method, health-related quality of life is measured by using standard questionnaires and adjusted for a 1-year period. A Markov model was used to assess the cost and outcome of SNM, BonT-A and OMT as comparators in patients with refractory $O A B$ failing on conservative management and first-line OMT (Fig. 1). In these clinical pathways, patients who have failed OMT will have three treatment pathways: (1) BonT-A injections; (2) the subsequent OMT line; and (3) SNM (contingent on a testing phase).

The model uses 9-month Markov cycles for BonT-A and all future direct medical costs and outcomes were discounted at $3 \%$ per annum with a 10 -year time horizon. A probabilistic sensitivity analysis using Monte Carlo Simulation with a cohort of 1000 patients was used to assess the uncertainty. The incremental cost-effectiveness ratio (ICER) of comparative therapies is presented by scatter plots on an ICER plane and cost-effectiveness acceptability curves.

The model assumes that all patients have equal access to all therapy options. Once a patient opts for a treatment path, they enter the Markov sub-model with the assumption that the patient will remain in the Markov state if therapy is successful. The SNM pathway starts with a test (SNM-T stage) to determine eligibility (Fig. 2). Success of the test enables the patient to proceed towards the SNM long-term therapy and the patient may remain on the SNM therapy unless it fails, which they may then opt for other treatment options. When a patient fails a therapy, the model allows for a switch to an alternative therapy.

$\mathrm{OAB}$ improvement is defined as $\geq 50 \%$ reduction in main incontinence or urgency frequency symptoms. For BonT-A, symptoms generally return within 9 months of treatment, hence frequent retreatment is needed to remain aligned with efficacy rates of randomized clinical trials (RCT). Patients with ambulatory BonT-A are assumed to be $20 \%$ of all patient populations. The wash out period, which refers to the interval in-between failure of BonT-A and the next successive treatment, is 4 months. The extrapolation of the shortterm outcomes from RCTs and other clinical studies up to 5 to 10 years were modelled together with different utility values associated to the models' health states. For SNM, short-term efficacy data for the implanted device (InterStim, Medtronic of Canada, Inc.) were obtained with the new tined lead procedure. Since long-term follow-up was not available for this new technology, the efficacy curve was constructed with the older technologies. The SNM success rate is $80 \%$ 


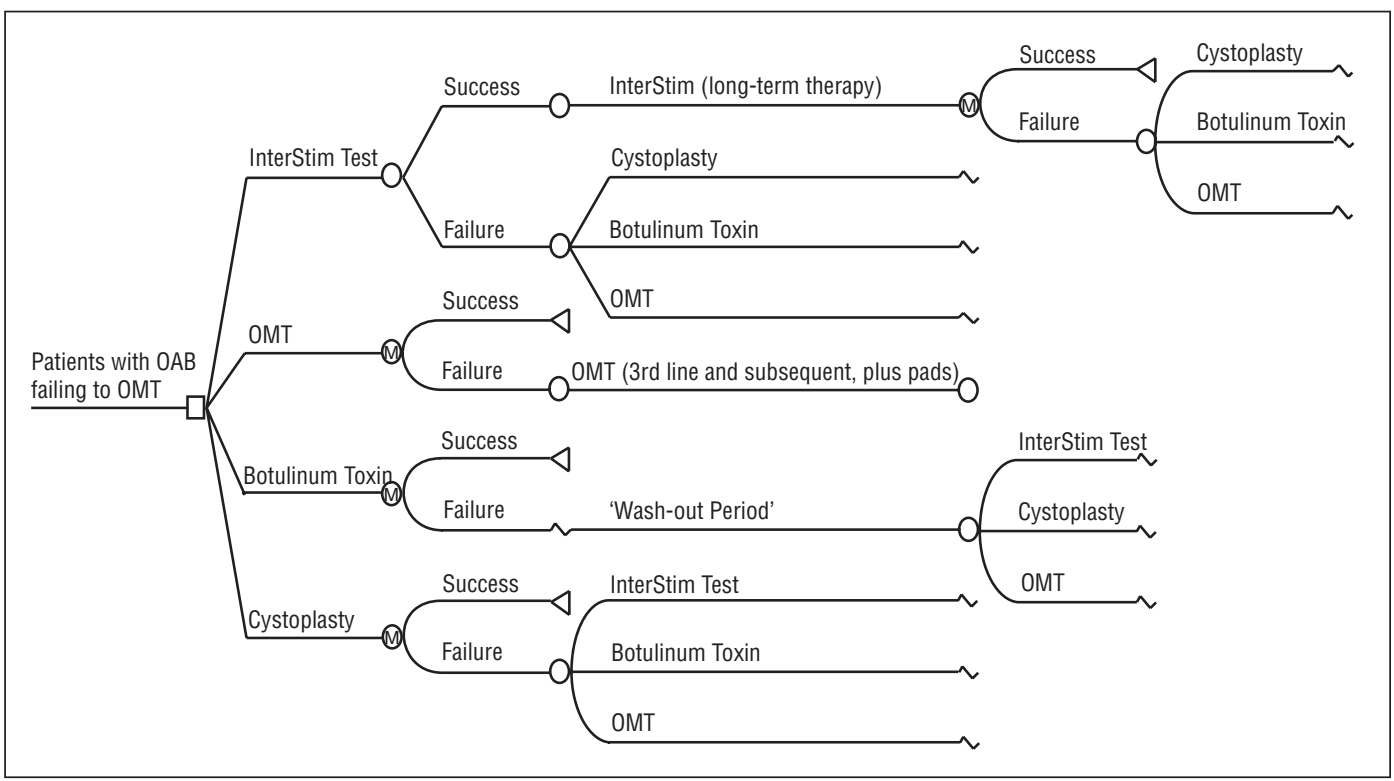

Fig. 2. Decision analytic tree.

with a 1- to 2-week time period to observe the SNM eligibility test. Once implanted, the device will be replaced about every 7 years. For SNM and BonT-A, the model assumes the patient has undergone pre-surgical procedures (i.e., physical and diagnostic tests).

\section{Model parameters}

Efficacy declined at a moderate rate $(0-15 \%$ range) from $90 \%$ at year 1 to $75 \%$ at year 10 . BonT-A treatment success rate declined more rapidly (range: $0-30 \%$ ) from $80 \%$ at year 1 to $50 \%$ at year 10 . OMT treatment rate remained the lowest and static at $4 \%$ throughout the treatment period (Table 1). The annual dropout rate, which may be attributed to adverse events or lack of efficacy, is defined as $7.5 \%$ for SNM and $2 \%$ for BonT-A.
Probability values for urinary tract infection for BonT-A, OMT, and SNM were $23 \%, 12 \%$, and $0 \%$ respectively (Table 2). Device-specific adverse events included implant site infection, generator/lead replacement and were at 3\%. The model assumes that $86 \%$ of patients who fail SNM will opt for BonT-A and 14\% would opt for OMT. Comparably, $80 \%$ of patients who fail BonT-A would opt for SNM and $20 \%$ for OMT.

\section{Cost inputs}

Healthcare resource utilization and associated direct medical costs from a Canadian provincial healthcare system were used in the model. The sources of healthcare resources used included: procedure, medications, complications, staff, diagnostics, disposables, devices, and follow-ups. The SNM test

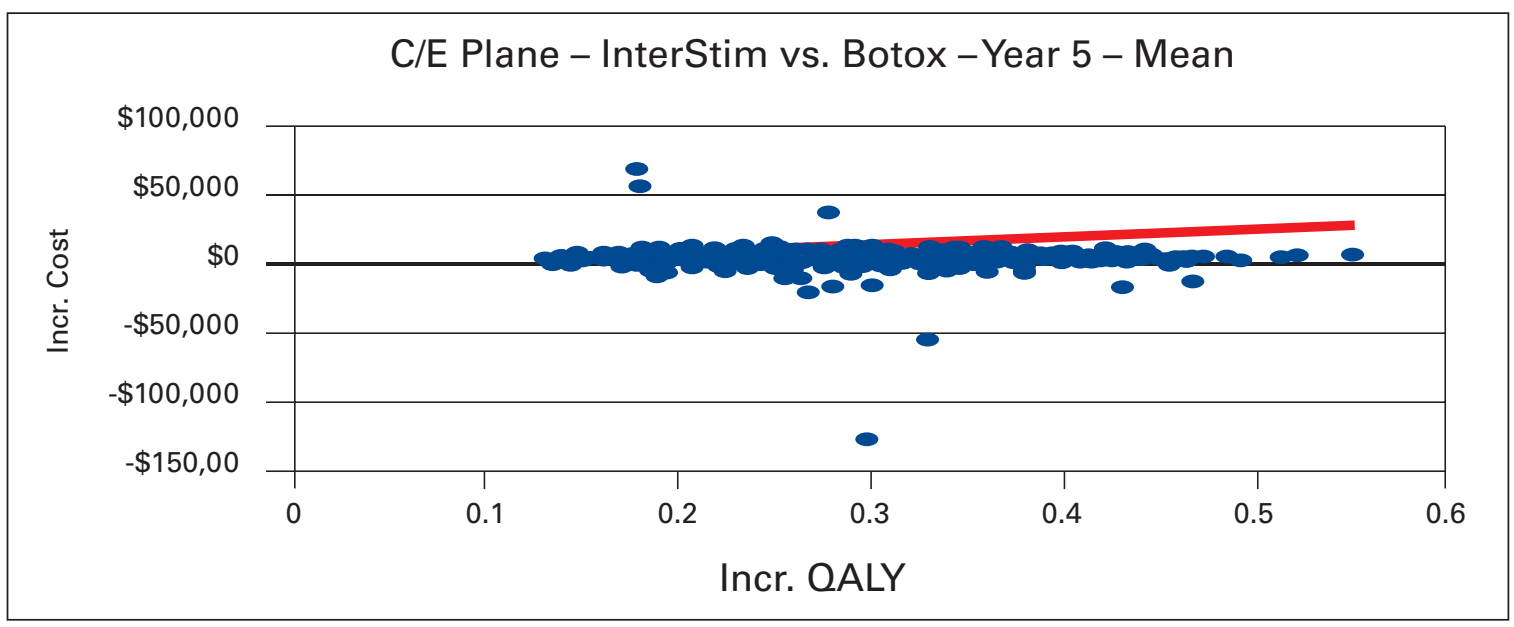

Fig. 3. Cost-effectiveness plane of sacral-neuromodulation and botulinum-toxin- $A$ at median range in year 5 . 


\begin{tabular}{lccc}
\hline \multicolumn{4}{l}{ Table 1. Treatment success rate of SNM, BonT-A and OMT } \\
\hline SNM & SNM & BonT-A & OMT \\
\hline 6 months & $80 \%$ & $80 \%$ & $4 \%$ \\
1 year & $90 \%$ & $80 \%$ & $4 \%$ \\
2 years & $86 \%$ & $74 \%$ & $4 \%$ \\
3 years & $82 \%$ & $68 \%$ & $4 \%$ \\
4 years & $78 \%$ & $63 \%$ & $4 \%$ \\
5 years & $75 \%$ & $59 \%$ & $4 \%$ \\
7 years & $75 \%$ & $54 \%$ & $4 \%$ \\
10 years & $75 \%$ & $50 \%$ & $4 \%$ \\
\hline
\end{tabular}

SNM: sacral-neuromodulation; BonT-A: botulinum-toxin-A; OMT: optimized medicaltherapy.

was conducted in an outpatient setting with relevant lab test (i.e., urinalysis and cystoscopy). Pending the success of the test stage, lab tests, such as chest radiography, prophylactic antibiotic and anesthesia, were considered prior to the SNM. The BonT-A pre-procedural stage would involve an outpatient visit and several lab tests (i.e., blood test, urodynamic, tuberculosis test). Drugs, such as antibiotics, and anesthesia may also be administered in addition to a psychiatric visit.

The model assumed that both SNM and BonT-A injections were performed in a hospital operating room at an estimated hourly rate for inpatient stay and for post-procedure. After failure of SNM treatment both at the test phase and treatment phase, costs included outpatient visits, analgesic drugs, nurse visits, pad, or psychiatric visits. Similarly, cost after BonT-A treatment failure can consist of several outpatient visits, urinalysis, pads, and flowmetry. Cost for OMT was limited to the cost of the drug administration for each treatment line. All costs are presented in 2014 Canadian dollars.

\section{Sensitivity analysis}

Uncertainty was evaluated with a second-order Monte Carlo simulation with 1000 random iterations simulating target

\begin{tabular}{|c|c|c|c|}
\hline Adverse events & SNM & BonT-A & OMT \\
\hline Re-operation & $8 \%$ & & \\
\hline Device Infection & $3 \%$ & & \\
\hline Urinary tract infection & & $23 \%$ & $12 \%$ \\
\hline Xerostomia & & & $16 \%$ \\
\hline Blur & & & $3 \%$ \\
\hline Constipation & & & $7 \%$ \\
\hline Change device - lead & $3 \%$ & & \\
\hline Change device - IPG & $3 \%$ & & \\
\hline Risk of femoral neck fracture & & & $8 \%$ \\
\hline
\end{tabular}

patient populations and a probabilistic sensitivity analysis. This technique is widely used as a means of converting uncertainties surrounding input parameters into probabilistic distributions. ${ }^{21,22}$ In analyzing the probability of cost-effectiveness, acceptability curves were diagramed to determine the degree of probability.

\section{Results}

The cost-effective analysis over a 10-year period demonstrated that SNM became cost-effective when compared to BonT-A during the 5-year treatment period (Table 3). The incremental cost savings of SNM in this period was $\$ 2775$ (95\% confidence interval [CI] \$1701-\$3941). The added effectiveness was 0.24 QALYs with the corresponding ICER showing dominance in all ranges until the 10-year period.

In considering the acceptability curve and the threshold of $\$ 50000$ per unit increase of QALY, the data states a 93\% chance that the additional cost of SNM, when compared to BonT-A, is less than $\$ 50000$ per unit increase in QALY during the 5-year period (Table 4, Fig. 3). It is important to note that SNM initially gains an advantage during the 4-year period and dominates at the 5 -year mark. Analysis of SNM in

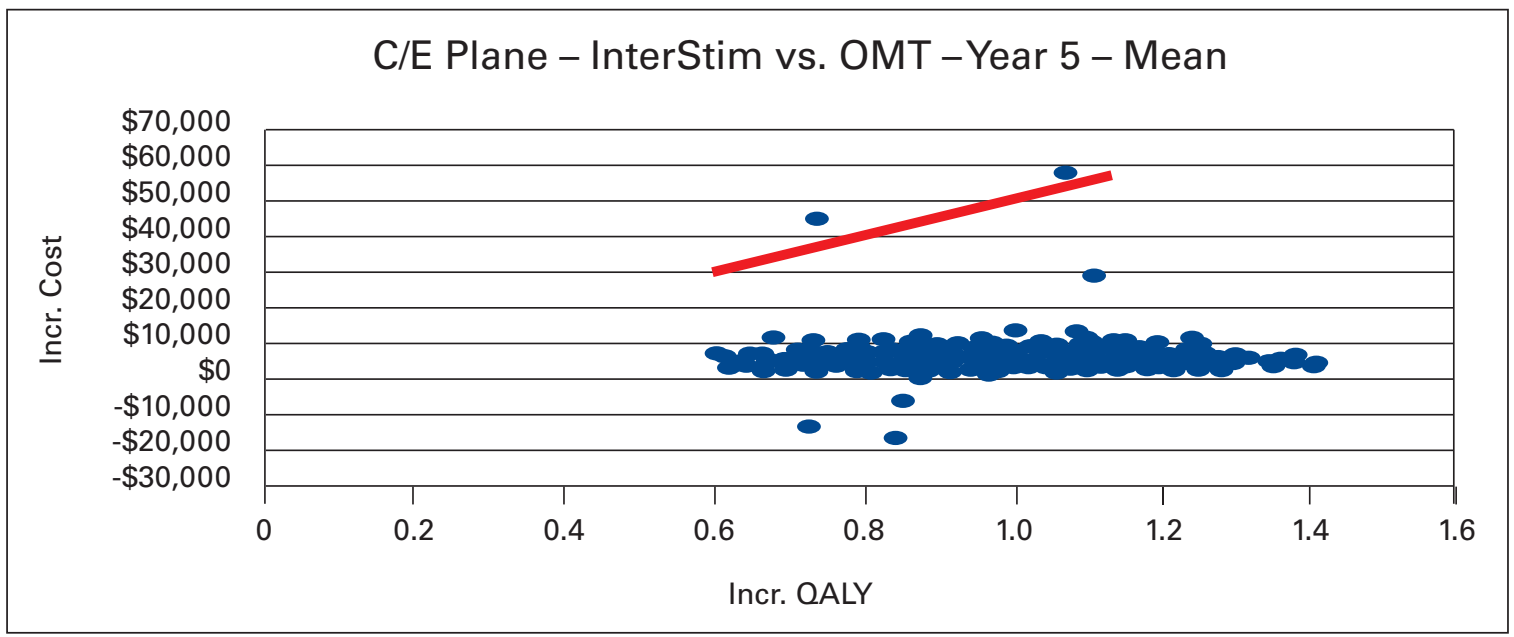

Fig. 4. Cost-effectiveness plane of sacral-neuromodulation vs. optimized medical-therapy at median range in year 5 . 
Table 3. SNM vs. BonT-A and OMT deterministic analysis

\begin{tabular}{|c|c|c|c|c|c|c|c|}
\hline \multicolumn{8}{|c|}{ SNM vs BonT-A } \\
\hline \multirow{2}{*}{ Year } & \multicolumn{3}{|c|}{$\Delta$ Cost } & $\triangle$ QALY & \multicolumn{3}{|c|}{ Cost/QALY } \\
\hline & Base Case & Low Range & High Range & Mean & Base case & Low Range & High Range \\
\hline 1 & $\$ 7237$ & $\$ 7574$ & $\$ 6709$ & 0.05 & $\$ 144067$ & $\$ 150769$ & $\$ 133558$ \\
\hline 2 & $\$ 4318$ & $\$ 4884$ & $\$ 3591$ & 0.09 & $\$ 44837$ & $\$ 50708$ & $\$ 37288$ \\
\hline 4 & $-\$ 651$ & $\$ 277$ & $-\$ 1691$ & 0.19 & SNM Dominant & $\$ 1436$ & SNM Dominant \\
\hline 5 & $-\$ 2775$ & $-\$ 1701$ & $-\$ 3941$ & 0.24 & \multirow{2}{*}{\multicolumn{3}{|c|}{ SNM Dominant }} \\
\hline 10 & $-\$ 9402$ & $-\$ 7698$ & $-\$ 11129$ & 0.51 & & & \\
\hline \multicolumn{8}{|c|}{ SNM vs. OMT } \\
\hline \multirow{2}{*}{ Year } & & $\Delta$ Cost & & $\triangle$ QALY & & Cost/QALY & \\
\hline & Base Case & Low Range & High Range & Mean & Base Case & Low Range & High Range \\
\hline 1 & $\$ 8878$ & $\$ 8812$ & $\$ 9008$ & 0.19 & $\$ 45999$ & $\$ 45655$ & $\$ 46672$ \\
\hline 2 & $\$ 5888$ & $\$ 5847$ & $\$ 6029$ & 0.38 & $\$ 15130$ & $\$ 15024$ & $\$ 15491$ \\
\hline 4 & $\$ 348$ & $\$ 335$ & $\$ 523$ & 0.76 & $\$ 455$ & $\$ 438$ & $\$ 684$ \\
\hline 5 & $-\$ 2233$ & $-\$ 2236$ & $-\$ 2039$ & 0.94 & \multirow{2}{*}{\multicolumn{3}{|c|}{ SNM Dominant }} \\
\hline 10 & $-\$ 11447$ & $-\$ 11347$ & $-\$ 11246$ & 1.76 & & & \\
\hline
\end{tabular}

QALY: quality-adjusted life years; SNM: sacral-neuromodulation; BonT-A: botulinum-toxin-A; OMT: optimized medical-therapy.

comparison with OMT after 10 years reveals that SNM is definitively cost-effective at the 2-year period and dominant at 5 and 10 years. The incremental cost of SNM during the 5 -year period is $-\$ 2233(95 \% \mathrm{Cl}-\$ 2,236--\$ 2,039)$, with an added effectiveness of 0.94 QALYs per patient during the 5 -year period. Additionally, the corresponding ICER shows dominance in all ranges from the 5 - to 10 -year period. With regard to the acceptability curve, when given the data, there is a $99 \%$ chance that the additional cost of SNM, when compared with OMT, is less than $\$ 50000$ per unit increase in QALY during the 5-year period (Fig. 4).

\section{Discussion}

There are different options for treating OAB. Standard pharmacological treatment includes anticholinergic medication or beta agonists to reduce bladder overactivity. Patients who are intolerant to the oral pharmacological treatment are usually offered intravesical injection of BonT-A toxin or SNM. In this study we assessed the economic impact of SNM when compared to BonT-A and oral anticholinergic drugs. The primary outcome measure was QALY, which was developed to measure health effectiveness, particularly cost-effectiveness. In the presence of scarce resources, the QALY may help decision makers in resource allocation. In North America and Europe, the QALY has been used as a standardized methodological approach to promote comparability in cost-effectiveness analysis of different healthcare products. ${ }^{23}$ We applied a Markov model to assess the cost and outcome of SNM, BonT-A and OMT in patients with refractory $\mathrm{OAB}$.

The Markov models have been used in health service decision making, including clinical and epidemiological application. One of the major strengths of the Markov model is the way it simply and intuitively handles both cost and outcomes of different treatment processes. We have applied the Markov model for the three modalities of treatment in overactive bladder with a 10-year time horizon.

A $50 \%$ or more improvement in OAB symptoms was the improvement outcome; this percentage has been adopted by different health educators.

The cost-effective analysis over a 10-year period showed that SNM becomes cost-effective and dominated in comparison to BonT-A during the first 5 years. The cost of SNM initially surpassed the BonT-A within the first 3 to 5 years. However, the acceptability curve and the threshold of $\$ 50000$ per unit increase of QALY, that a 93\% chance of an additional cost of SNM when compared with BonT-A, is less than \$50 000 per unit increase in QALY during the 5 year period (Fig. 3). Our Markov model clearly showed costeffectiveness since there was a 99\% chance of an additional cost of SNM when compared with OMT; our threshold was less than 50000 per unit increase in QALY during the 5 year period (Fig. 4).

SNM treatment is more cost effective after the 2 year mark when compared to OMT and dominant after the 5 year period when compared with both BonT-A and OMT. The sensitivity analysis further demonstrates the effectiveness and validity of SNM in comparison to the alternatives. The longterm side-effects however have not been noted and further testing would be required to assess if any long-term side effects may be prevalent. Lastly, the demographic formation of the test subjects with respect to age would contribute to further testing to gain knowledge of cost-effectiveness in specific ages should be conducted.

Similar to other economic evaluation of technologies, this study has some limitations. In the absence of direct longterm comparison between therapies, some assumptions were 


\begin{tabular}{lccc}
\hline Table 4. SNM vs. BonT-A and OMT probabilistic analysis \\
\hline SNM vs. BonT-A & \multicolumn{3}{c}{$\%<$ Cost-effective threshold } \\
\hline \multirow{3}{*}{1 year } & Mean & Low range & High range \\
\cline { 2 - 4 } 2 years & $0.50 \%$ & $0.10 \%$ & $0.40 \%$ \\
4 years & $26.70 \%$ & $21.60 \%$ & $48.60 \%$ \\
5 years & $94.40 \%$ & $95.60 \%$ & $93.90 \%$ \\
10 years & $93.20 \%$ & $94.60 \%$ & $89.40 \%$ \\
\hline SNM vs. OMT & $85.80 \%$ & $88.60 \%$ & $77.70 \%$ \\
\hline & \multicolumn{2}{c}{$\%<$ Cost-effective threshold } \\
1 year & Mean & Low range & High range \\
2 years & $17.90 \%$ & $22.00 \%$ & $9.40 \%$ \\
4 years & $99.90 \%$ & $99.80 \%$ & $100.00 \%$ \\
5 years & $99.60 \%$ & $99.60 \%$ & $100.00 \%$ \\
10 years & $99.60 \%$ & $99.60 \%$ & $100.00 \%$ \\
\hline
\end{tabular}

QALY: quality-adjusted life years; SNM: sacral-neuromodulation; BonT-A: botulinumtoxin-A; OMT: optimized medical-therapy.

made which poses limitation in this study. In this study the efficacy, utility scores and annual drop-out rates were constant, which may not represent real practice. Another major limitation is the use of generic quality of life questionnaires, which may not accurately measure the psychological impact of OAB. This study used the provincial payer perspective; however, using a broader societal perspective might be more appropriate as it may represent the many challenges and limitations patient with $\mathrm{OAB}$ face. Another limitation of this study is the ability to extrapolate the results between jurisdictions. Therapy pathways, healthcare and procedural costs and expertise may vary significantly between healthcare systems.

\section{Conclusion}

This study demonstrates that SNM is a cost-effective treatment option to manage patients with refractory $O A B$ when compared to either BonT-A or OMT. From a Canadian payers' perspective, SNM may be considered a first-line treatment option with superior long-term outcomes.

Acknowledgement: We thank our advisory board: J. Gajewski, R. Magalie, J. Corcos and L. Tu.

Competing interests: Dr. Hassouna is a member of the Advisory Board for Medtronic of Canada. Mr. Sadri is an employee of Medtronic of Canada.

This paper has been peer-reviewed.

\section{References}

1. Radomski SB, Barkin, J. Medical management of overactive bladder. Can J Urol 2012;19(Suppl 1):2-9.

2. Stewart W, Van Rooven J, Cundiff $G$, et al. Prevalence and burden of overactive bladder in the United States. World J Urol 2003;20:327-36.

3. Barkin J. Overactive bladder. Can J Urol 2011;18(Suppl 1):8-13.

4. Siegel WS, Catanzaro F, Dijkema EH, et al. Long-term results of multicenter study on sacral nerve stimulation for the treatment of urinary urge incontinence urgency/frequency and retention. Urology 2000;56(Suppl 1):87-91.

5. Herschorn S, Gajewski J, Schulz J, et al. A population-based study of urinary symptoms and incontinence: The Canadian Urinary Bladder Survey. BJU Int 2008;101:52-8.

6. Chapple C, Gormley AE. Developments in pharmacological therapy for the overactive bladder. BJU Int 2006;98 (Suppl 1):78-87. http://dx.doi.org/10.1111/i.1464-410X.2006.06381.x

7. Coyne SK, Sexton CC, Irwin ED, et al. The impact of overactive bladder, incontinence and other lower urinary tract symptoms on quality of life, work productivity, sexuality and emotional well-being in men and women: results from the EPIC study. BJU Int 2008;101:1388-95. http://dx.doi.org/10.1111/j.1464410X.2008.07601.x

8. Arnold J, McLeod N, Thani-Gasalam R, et al. Overactive bladder syndrome- management and treatment options. Aust Fam Physician 2012; 41:878-83.

9. Irwin ED, Mungapen L, Milsom I, et al. The economic impact of overactive bladder syndrome in six Western countries. BJU Int 2009;103:202-9. http://dx.doi.org/10.1111/i.1464-410X.2008.08036.x

10. Hu WT, Wagner HT, Bentkover DJ, et al. Costs of urinary incontinence and overactive bladder in the United States: A comparative study. Urology 2004;63:461-5. http://dx.doi.org/10.1016/i.urology.2003.10.037

11. Geoffrion R. Treatments for overactive bladder: Focus on pharmacotherapy. J Obstet Gynaecol Can 2012;34:1092-101.

12. Andersson KE, Chapple C, Wein A. The basis for drug treatment of the overactive bladder. World I Urol 2001;19:294-8. http://dx.doi.org/10.1007/PL00007101

13. Andersson EK. Antimuscarinics for treatment of overactive bladder. Lancet Neurol 2004;3:46-53. http:// dx.doi.org/10.1016/S1474-4422(03)00622-7

14. Jensen D. Uninhibited neurogenic bladder treated with prazosin. Scand J Urol Nephrol 1981;15:229-33. http://dx.doi.org/10.3109/00365598109179608

15. Braverman SA, Ruggieri RM, Pontari AM. The M2 muscarinic receptor subtype mediates cholinergic bladder contractions in patients with neurogenic bladder dysfunction. Am J Physiol Regul Integr Comp Physiol 2004;165(Suppl):36.

16. Ohlstein EH, von Keitz A, Michel MC. A multicenter, double-blind, randomized, placebo-controlled trial of the b3-adrenoceptor agonist solabegron for overactive bladder. Eur Urol 2012;62:834-40. http:// dx.doi.org/10.1016/i.eururo.2012.05.053

17. Sahai A, Khan SM, Dasgupta P. (2007). Efficacy of botulinum toxin-A for treating idiopathic detrusor overactivity: Results from a single center, randomized, double-blind, placebo controlled trial. J Urol 2007;177:2231-6. http://dx.doi.org/10.1016/i.juro.2007.01.130

18. Schmid MD, Sauermann P, Werner M, et al. Experience with 100 cases treated with botulinum-A toxin injections in the detrusor muscle for idiopathic overactive bladder syndrome refractory to anticholinergic. J Urol 2006;176:177-85. http://dx.doi.org/10.1016/S0022-5347(06)00590-8

19. Dmochowski R, Chapple C, Nitti VW, et al. Efficacy and safety of onabotulinumtoxin A for idiopathic overactive bladder: A double-blind, placebo controlled, randomized, dose ranging trial. J Urol 2010;18:2416-22. http://dx.doi.org/10.1016/i.juro.2010.08.021

20. Marcelissen AT, Leong KR, de Bie AR, et al. Long-term results of sacral neuromodulation with the tined lead procedure. J Urol 2010;184:1997-2000. http://dx.doi.org/10.1016/i.juro.2010.06.142

21. Indinnimeo M, Ratto C, Moschella MC, et al. Sacral Neuromodulation for the Treatment of Fecal Incontinence: Analysis of Cost-Effectiveness. Dis Colon Rectum 2010;54:1661-9. http://dx.doi. org/10.1007/DCR.0b013e3181f46309

22. Leong KR, Wachter GGS, Joore AM, et al. Cost-effectiveness analysis of sacral neuromodulation and botulinum toxin A treatment for patients with idiopathic overactive bladder. BJU Int 2011;108:558-64. http://dx.doi.org/10.1111/j.1464-410X.2010.09905.x

23. Winstein M, Torrance G, Mcguire A. Qualys: The Basics. Value Health 2009;12:S5-9.

Correspondence: Dr. Magdy Hassouna, University Health Network, Suite 3-130, 610 University Ave., Toronto, ON M5G 2M9; magdy.hassouna@uhn.on.ca 